Supporting Information

\title{
Do the Evaluation Parameters Reflect Intrinsic Activity of Electrocatalysts in Electrochemical Water Splitting?
}

\section{Sengeni Anantharaj ${ }^{*,+,+}$ and Subrata Kundu $u^{*,+,}$}

†Academy of Scientific and Innovative Research (AcSIR), Ghaziabad - 201 002, Uttar Pradhesh, India.

Materials Electrochemistry Division (MED), CSIR-Central Electrochemical Research Institute (CECRI), Karaikudi - 630006, Tamil Nadu, India.

* To whom correspondence should be addressed, E-mail: kundu.subrata@gmail.com, skundu@cecri.res.in and anantharaj1402@gmail.com. Phone: (+ 91) 4565-241486 and (+ 91) 4565-241487. 


\section{EXPERIMENTAL}

\section{Methods and Materials}

$\mathrm{NiO}$ oxide powder catalyst was procured from Sigma-Aldrich. $\mathrm{Hg} / \mathrm{HgO}$ reference electrode was purchased from $\mathrm{CH}$ Intruments pvt. Ltd. Deionized water (18 $\mathrm{M} \Omega$ ) was used for the entire electrochemical characterizations. The $\mathrm{NiO}$ modified carbon cloth (CC) was used as working electrode and a blank carbon cloth with large geometrical surface area $(1.5 \times 3 \mathrm{~cm})$ was used as a counter electrode. $\mathrm{CH}$ Instrument model number $\mathrm{CHI} 6804 \mathrm{c}$ was used for entire electrochemical studies.

\section{Electrode Fabrications}

$\mathrm{CC}$ stripes of dimension $1 \mathrm{~cm} \times 5 \mathrm{~cm}$ were acid functionalized to make them hydrophilic and the same were then washed several times with ethanol and DI water before drying. The dried CC stripes were then used as substrate electrodes. A catalyst ink of composition $0.75 \mathrm{~mL}$ water, $0.2 \mathrm{~mL}$ 2-propanol and $0.05 \mathrm{~mL} \mathrm{5 \%} \mathrm{Nafion} \mathrm{solution} \mathrm{was} \mathrm{prepared} \mathrm{by} \mathrm{dispersing} \mathrm{exactly} 3 \mathrm{mg}$ of the $\mathrm{NiO}$ powder. This was sonicated for $20 \mathrm{~min}$ consecutively to homogenize the same. The homogenized ink was later drop casted on CC stripes within $1 \mathrm{~cm} 2$ area. The volume of coated ink was varied as $34.5,52.0,69.0,103.5$ and $138.0 \mu \mathrm{L}$ respectively to have the loadings of 0.205 , $0.310,0.410,0.615$ and $0.820 \mathrm{mg} \mathrm{cm}^{-2}$. The electrodes were then dried in dark at room temperature for overnight and then directly used as working electrodes.

\section{Electrochemical Characterizations}

All electrochemical characterizations were carried out in $1 \mathrm{M} \mathrm{KOH}$ with a conventional three electrode electrochemical system in which $1 \mathrm{M} \mathrm{KOH}$ filled $\mathrm{Hg} / \mathrm{HgO}$ reference electrode was used as a reference electrode with an acid treated CC stripe of large surface area as a counter electrode. All linear sweep voltammograms (LSVs) were recorded at $5 \mathrm{mV} \mathrm{s}^{-1}$ scan rate and 
compensated for iR drop for $100 \%$ wherever required. Electrochemical impedance spectroscopy (EIS) analysis was carried out $1.6 \mathrm{~V}$ vs. RHE with an AC amplitude of $5 \mathrm{mV}$.

\section{Determination of Number of Ni Sites Involved in OER Based on Redox Peak Method} For NiO/CC-0.205 $\mathrm{mg} \mathrm{cm}^{-2}$ :

$$
\begin{array}{ll}
\text { Area under NiOOH formation peak } & =4.465 \times 10^{-5} \mathrm{~A} \mathrm{~V} \\
\text { Charge associated with NiOOH formation } & =4.465 \times 10^{-5} \mathrm{~A} \mathrm{~V} / 0.005 \mathrm{~V} \mathrm{~s}^{-1} \\
& =8.93 \times 10^{-3} \mathrm{~A} \mathrm{~s} \\
& =8.93 \times 10^{-3} \mathrm{C} \\
\text { Charge of an electron } & =1.602 \times 10^{-19} \mathrm{C}
\end{array}
$$

Hence,

$$
\begin{aligned}
\text { Number of electrons transferred } & =8.93 \times 10^{-3} \mathrm{C} / 1.602 \times 10^{-19} \mathrm{C} \\
& =5.57 \times 10^{16}
\end{aligned}
$$

Since, the $\mathrm{NiO} / \mathrm{Ni}(\mathrm{OH})_{2}$ to $\mathrm{NiOOH}$ formation reaction is a single electron transfer reaction, the number of electron transferred during the reaction is exactly equal to the number of $\mathrm{Ni}$ sites involved in the reaction.

Hence,

Number of Ni sites that actually involved in OER $\quad=5.57 \times 10^{16}$

\section{Determination of Turnover Frequency (TOF)}

TOF at $1.8 \mathrm{~V}$ vs. RHE for $\mathrm{NiO} / \mathrm{CC}-0.205 \mathrm{mg} \mathrm{cm}^{-2}$ is

$$
\begin{aligned}
\text { TOF } & =0.0387 \mathrm{~A} \times 6.023 \times 10^{23 / 4} \times 96485 \mathrm{C}(\text { or A s }) \times 5.57 \times 10^{16} \\
& =8.99 \times 10^{-1} \mathrm{~s}^{-1}
\end{aligned}
$$



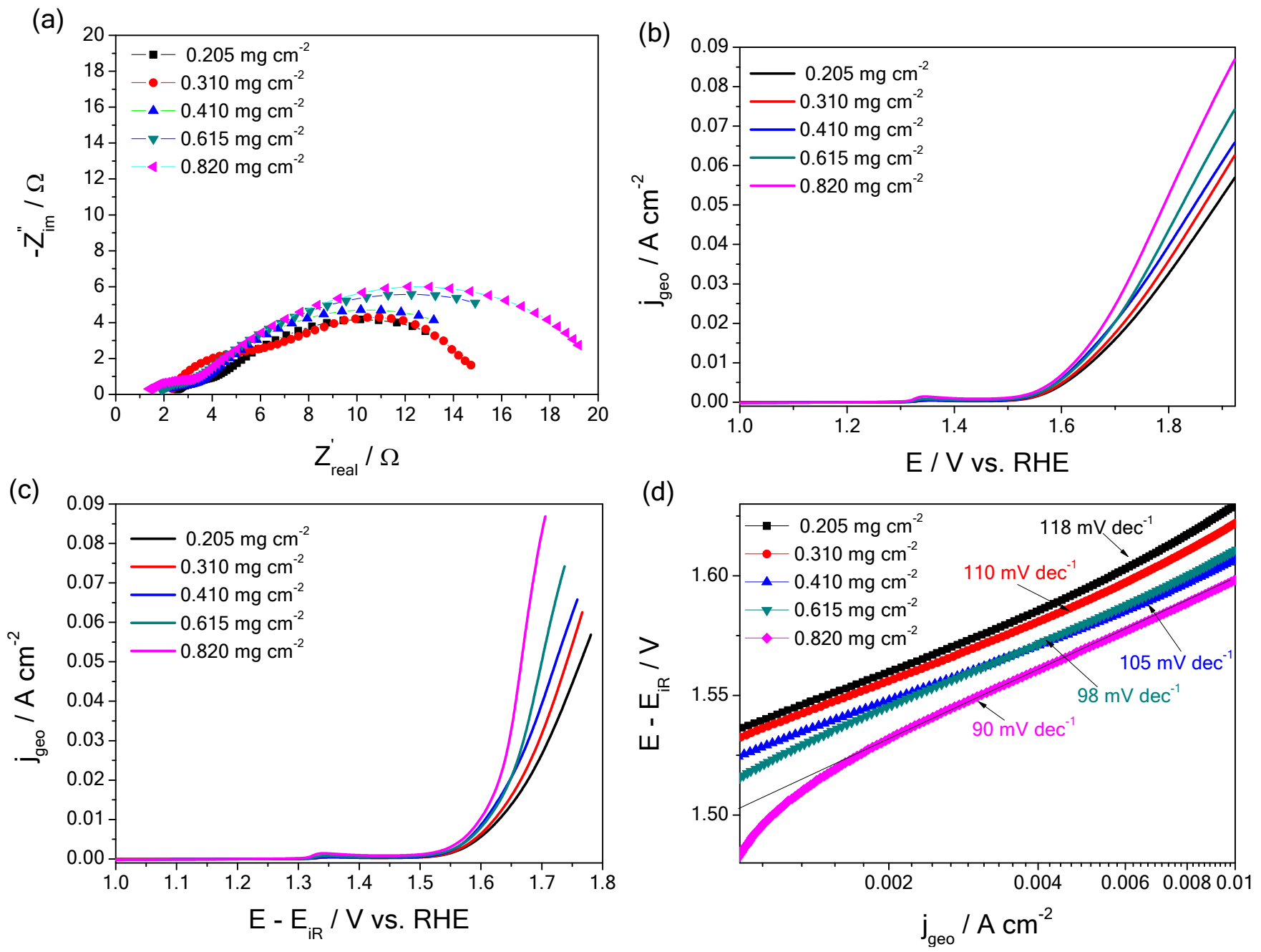

Figure S1: (a) Nyquist plots of the studied $\mathrm{NiO} / \mathrm{CC}$ electrodes of increasing loading. (b and c) iR drop uncompensated and compensated LSVs of the same. (d) The corresponding Tafel plots. 

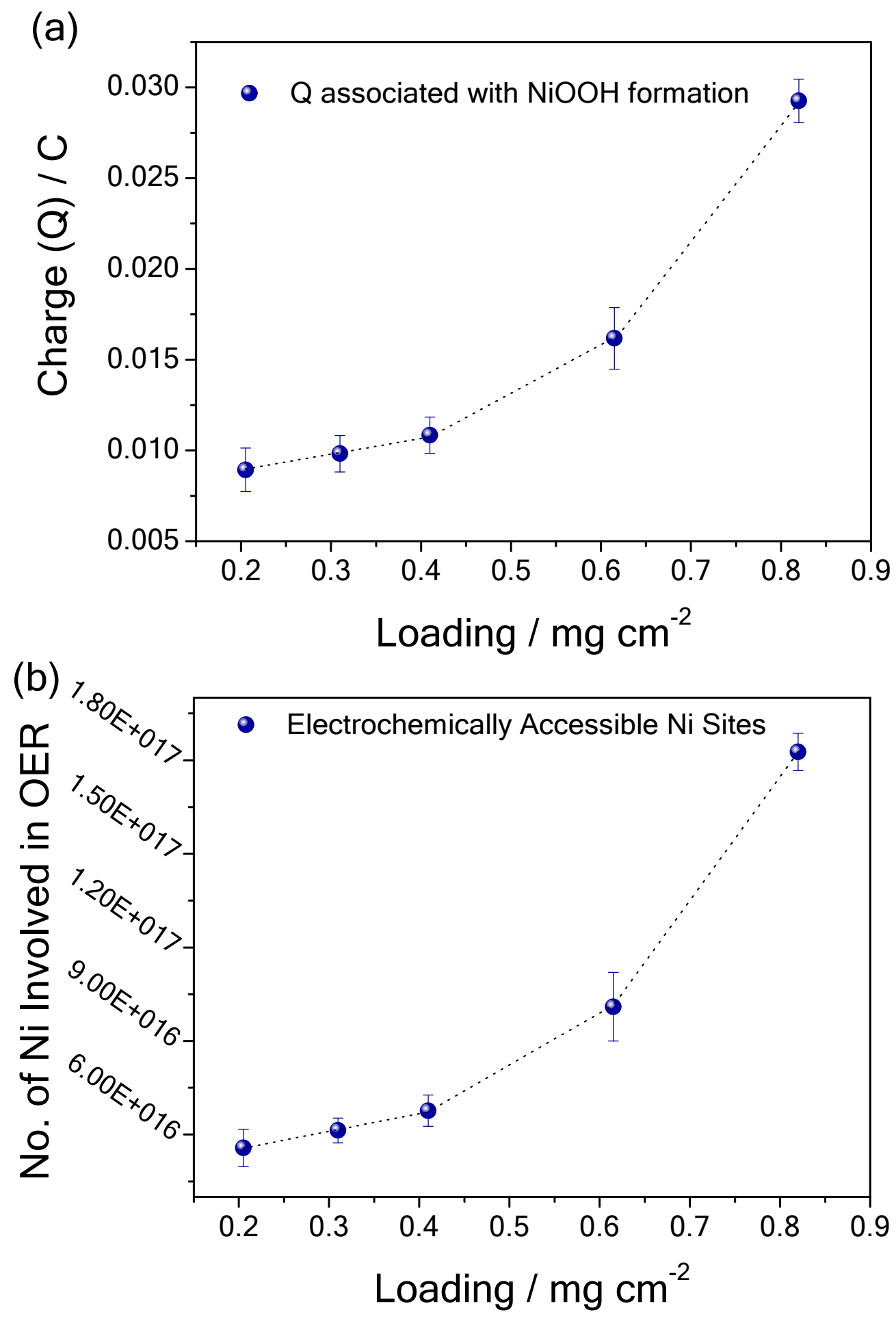

Figure S2: (a) plot of loading vs. charge associated with NiOOH formation. (b) plot of loading vs. number of available Ni sites. 


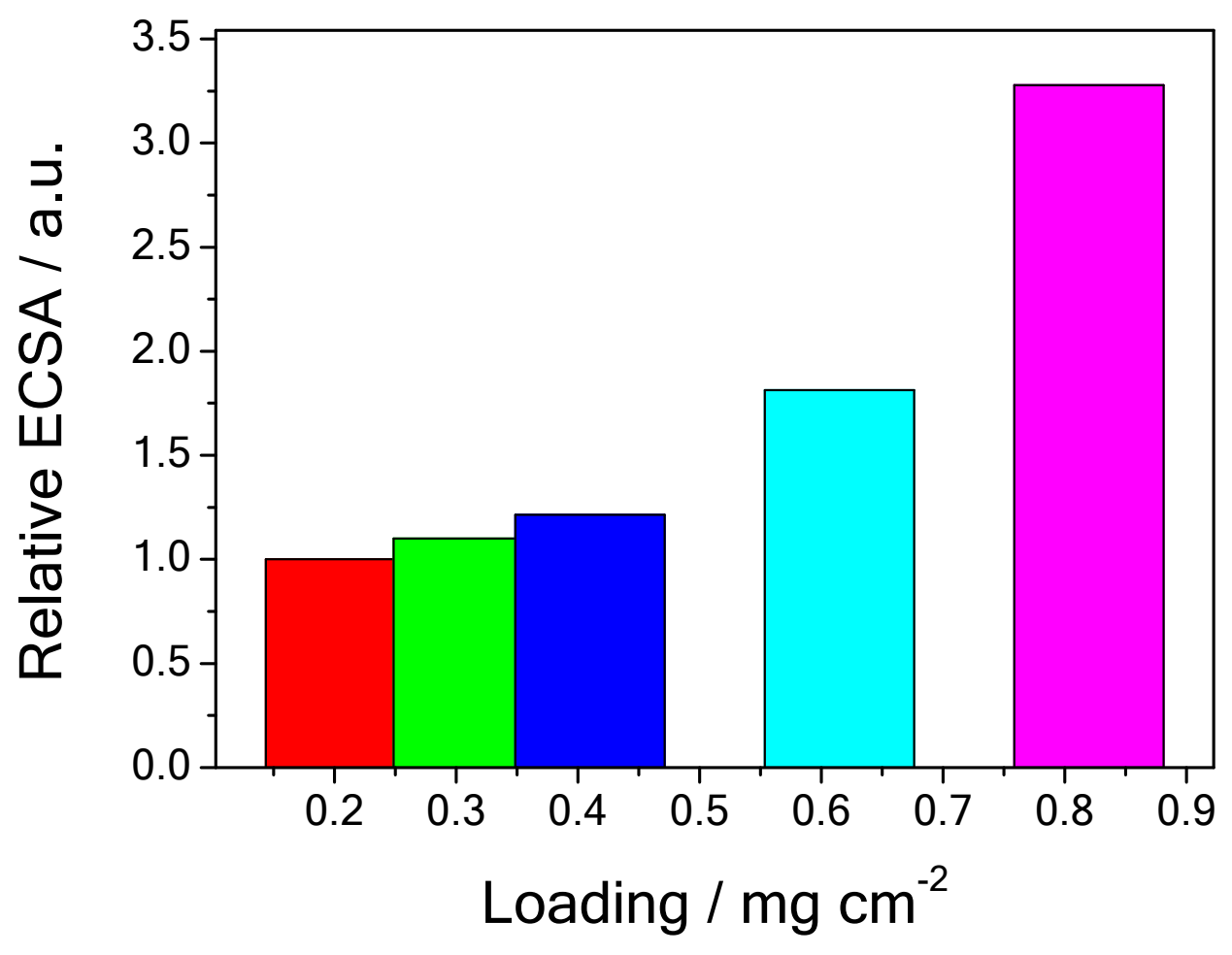

Figure S3: Plot of loading vs. relative ECSA of all studied $\mathrm{NiO}$ catalytic interfaces in $1 \mathrm{M} \mathrm{KOH}$. 
Table S1: Results of the electrocatalytic study.

\begin{tabular}{|c|c|c|c|c|}
\hline $\begin{array}{l}\text { NiO Loading } \\
\left(\mathrm{mg} \mathrm{cm}^{-2}\right)\end{array}$ & 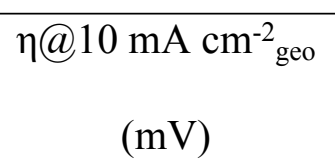 & $\begin{array}{l}\text { Tafel Slope } \\
\left(\mathrm{mV} \mathrm{dec}^{-1}\right)\end{array}$ & $\begin{array}{c}\eta @ 10 \mathrm{~mA} \mathrm{~cm}^{-2} \mathrm{ECSA} \\
(\mathrm{mV})\end{array}$ & $\begin{array}{c}\text { TOF } \\
\left(\times 10^{-1} \mathrm{~s}^{-1}\right)\end{array}$ \\
\hline 0.205 & $426 \pm 5$ & $118 \pm 4$ & $423 \pm 3$ & 8.99 \\
\hline 0.310 & $416 \pm 5$ & $110 \pm 2$ & $422 \pm 5$ & 8.96 \\
\hline 0.410 & $403 \pm 4$ & $105 \pm 3$ & $420 \pm 6$ & 8.93 \\
\hline 0.615 & $403 \pm 9$ & $98 \pm 6$ & $457 \pm 5$ & 6.85 \\
\hline 0.820 & $390 \pm 6$ & $90 \pm 3$ & $504 \pm 6$ & 4.43 \\
\hline
\end{tabular}

